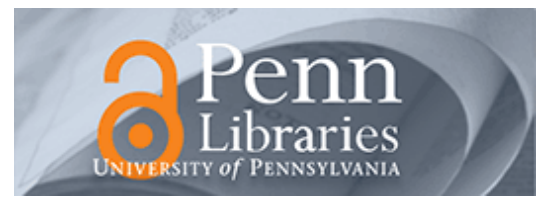

University of Pennsylvania ScholarlyCommons

$11-3-2008$

\title{
High-resolution characterization of defects in oxide thin films
}

\author{
Maxim N. Nikiforov \\ University of Pennsylvania, maximn@seas.upenn.edu \\ Matthew J. Brukman \\ University of Pennsylvania, mbrukman@seas.upenn.edu \\ Dawn A. Bonnell \\ University of Pennsylvania, BONNELL@SEAS.UPENN.EDU
}

Follow this and additional works at: https://repository.upenn.edu/mse_papers

\section{Recommended Citation}

Nikiforov, M. N., Brukman, M. J., \& Bonnell, D. A. (2008). High-resolution characterization of defects in oxide thin films. Retrieved from https://repository.upenn.edu/mse_papers/165

Copyright (year) American Institute of Physics. This article may be downloaded for personal use only. Any other use requires prior permission of the author and the American Institute of Physics.

Reprinted from:

High-resolution characterization of defects in oxide thin films Maxim N. Nikiforov, Matthew J. Brukman, and Dawn

A. Bonnell, Appl. Phys. Lett. 93, 182101 (2008), DOI:10.1063/1.2982082

Publisher URL: http://link.aip.org/link/?APPLAB/93/182101/1

This paper is posted at ScholarlyCommons. https://repository.upenn.edu/mse_papers/165

For more information, please contact repository@pobox.upenn.edu. 


\title{
High-resolution characterization of defects in oxide thin films
}

\author{
Abstract \\ Nanometer sized defects in thin HfOx films are detected by atomic force microscopy facilitated leakage \\ current measurements. Differences in the electrical properties of individual defects were distinguished. \\ The effects of two mechanisms that localize the tip-sample interaction and increase spatial resolution \\ were calculated. The expected increase in tip-sample current due to stress induced phase \\ transformations and band gap narrowing has been calculated, and a behavior diagram is presented that \\ shows the pressure necessary to generate a detectable current increase as a function of tip radius.

\section{Keywords} \\ SCANNING CAPACITANCE MICROSCOPY, SPREADING RESISTANCE MICROSCOPY, SPATIAL- \\ RESOLUTION, SILICON, SI, atomic force microscopy, dielectric thin films, flaw detection, leakage currents \\ Comments \\ Copyright (year) American Institute of Physics. This article may be downloaded for personal use only. Any \\ other use requires prior permission of the author and the American Institute of Physics. \\ Reprinted from: \\ High-resolution characterization of defects in oxide thin films Maxim N. Nikiforov, Matthew J. Brukman, \\ and Dawn A. Bonnell, Appl. Phys. Lett. 93, 182101 (2008), DOI:10.1063/1.2982082 \\ Publisher URL: http://link.aip.org/link/?APPLAB/93/182101/1
}




\title{
High-resolution characterization of defects in oxide thin films
}

\author{
Maxim N. Nikiforov, ${ }^{\text {a) }}$ Matthew J. Brukman, and Dawn A. Bonnell ${ }^{\text {b) }}$ \\ Department of Materials Science and Engineering, University of Pennsylvania, Philadelphia, \\ Pennsylvania 19104, USA
}

(Received 22 May 2008; accepted 24 August 2008; published online 3 November 2008)

\begin{abstract}
Nanometer sized defects in thin $\mathrm{HfO}_{x}$ films are detected by atomic force microscopy facilitated leakage current measurements. Differences in the electrical properties of individual defects were distinguished. The effects of two mechanisms that localize the tip-sample interaction and increase spatial resolution were calculated. The expected increase in tip-sample current due to stress induced phase transformations and band gap narrowing has been calculated, and a behavior diagram is presented that shows the pressure necessary to generate a detectable current increase as a function of tip radius. (C) 2008 American Institute of Physics. [DOI: 10.1063/1.2982082]
\end{abstract}

As microelectronics fabrication processes achieve an ever-decreasing device size, reducing the number and size of defects therein becomes increasingly critical. For example, oxide films in transistors are of the order of $1 \mathrm{~nm}$ thick and defects of atomic dimensions can be fatal. These size scales are particularly relevant to the emerging use of high $\kappa$ films. This situation represents the ultimate challenge in identifying and characterizing property variations at the smallest length scale. Scanning probe microscopy offers high spatial resolution in the characterization of a variety of electronic properties. ${ }^{1}$ Scanning spreading resistance microscopy $(\mathrm{SSRM})^{2}$ and scanning capacitance microscopy (SCM) are two primary techniques that probe local properties, utilizing a tip that is in contact with a surface. ${ }^{3}$ Recently, lateral resolution approaching $1 \mathrm{~nm}$ has been achieved with $\mathrm{SCM}^{4}$ in imaging of localized electronic states near a $\mathrm{SiO}_{2}$ surface in ultrahigh vacuum. Spatial resolution in the $<5 \mathrm{~nm}$ range was demonstrated for SSRM in air on InGaAs quantum well structures. ${ }^{5}$ Later $1-3 \mathrm{~nm}$ reproducibility was demonstrated on $p$ - and $n$-metal-oxide-semiconductor structures. ${ }^{6}$

While this spatial resolution is encouraging, it is counterintuitive that resolution a factor of 10 smaller than the tip dimension is possible. Finite tip dimensions usually act to increase rather than decrease apparent dimensions. Furthermore, the applicability in terms of range of materials has not been explored nor has the potential to map and distinguish differences in defects been demonstrated. This letter presents localized conductance measurements of nanometer sized defects on $\mathrm{HfO}_{x}$ thin films, quantifies differences in defects that can be used to determine structural origins, and compares two mechanisms of tip-surface interactions that result in subtip-diameter spatial resolution.

A $12 \AA$ thick $\mathrm{HfO}_{x}$ thin film on Si (100) (dopant density is $\sim 10^{15}-10^{17} \mathrm{~cm}^{-3}$ ) was used as a model system to test the limits of defect detection and identification. Local conductance was measured using an ambient commercial atomic force microscope (AFM) (Veeco Dimension 3100, Nanoscope IVa controller) fitted with a high gain amplifier with a current detection range of $\pm 10 \mathrm{pA}$ and a $40 \mathrm{fA}$ white noise level. The sample was biased over a $\pm 1.5 \mathrm{~V}$ range and current between the grounded tip and sample was monitored.

\footnotetext{
${ }^{a)}$ Currently at Oak Ridge National Laboratory.

${ }^{b)}$ Electronic mail: bonnell@1rsm.upenn.edu.
}

$I-V$ curves were constructed by averaging selected areas of voltage dependent conductivity maps. PtIr-coated silicon tips with radii of curvature $35 \mathrm{~nm}$, as quoted by manufacturer attached to cantilevers with spring constants $1-5 \mathrm{~N} / \mathrm{m}$ (Nanosensors), were used in the experiment. The maximum roughness of $\mathrm{HfO}_{x}$ thin films was in the range of 1-2 $\AA$ for a scan size of $1 \times 1 \mu \mathrm{m}^{2}$.

First, maps of leakage current were acquired at constant sample bias; when defects were located (indicated by locally high current of either polarity) the AFM tip was moved to the area of interest and $I-V$ curves were acquired. The stability against thermal and mechanical drifts in ambient conditions was not sufficient to fully characterize $1 \mathrm{~nm}$ sized defects in point spectroscopy. As an alternative, sequential current maps were acquired over a range of biases, and $I-V$ curves at each point were reconstructed. The same drift is present when acquiring images; however, it is corrected with reference to topographical features.

Figure 1 shows current maps acquired in the $\pm 1.5 \mathrm{~V}$ range in several increments. The regions of increased current are attributed to defects in the film. Comparisons of $I-V$ properties constructed from current averaged over selected areas are made in Fig. 2, where (A) is the area over a positive defect, (B) is the area over a negative defect, and (C) is an area with no defect. On areas where no defects are apparent, the tip/sample junction exhibits classic tunneling behavior over the entire range of biases with current in the pA range. This is consistent with the presence of an ideal $\mathrm{HfO}_{x}$ with an approximately $6 \mathrm{eV}$ band gap. Over the defects, increased current at voltages less than $-1.25 \mathrm{~V}$ implies the presence of localized midgap states, in some cases above and some cases below the Fermi level. While the atomic structure of these defects is not determined here and the interaction of defects with the ambient is not excluded, it is interesting to note that defect energies determined from Fig. 2 of 2.4 and $4.4 \mathrm{eV}$ are consistent with those estimated for oxygen vacancies and oxygen interstitial atoms, respectively. Tip-induced oxidation is a known problem when Si surfaces are imaged in air, but the pre-existing hafnia coating serves as a barrier against oxidation. Furthermore, the voltages used are not sufficient to reduce the Hf within the coating.

The similarity of behaviors of area A at negative sample bias, area B at positive bias, and no defect area in the whole voltage range suggests that the conduction mechanism is the 


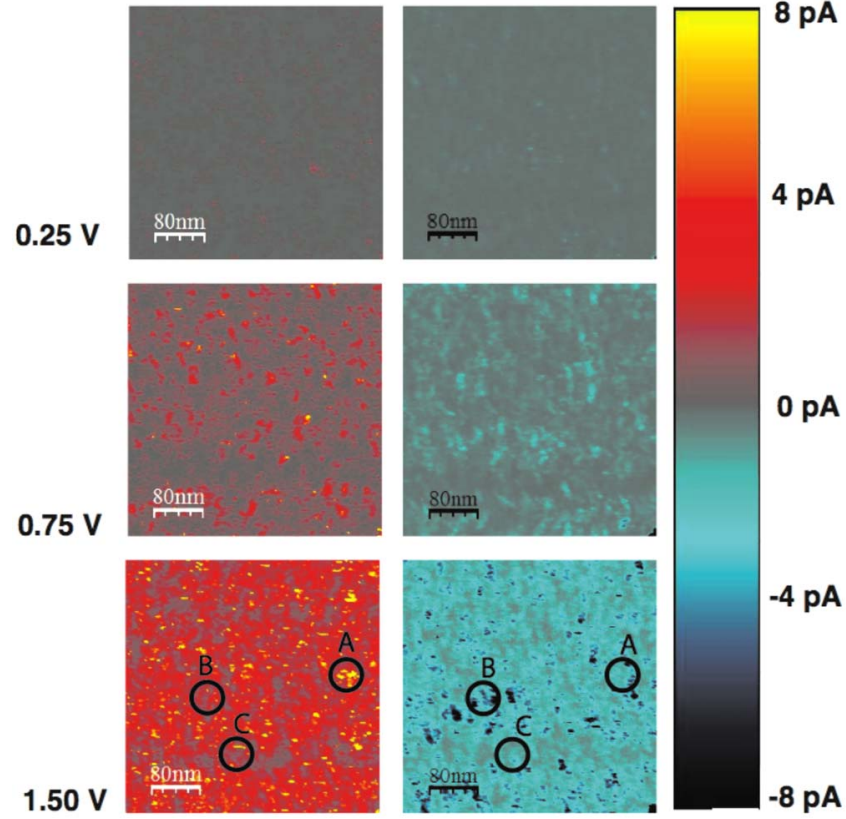

FIG. 1. (Color online) Current map of $\mathrm{HfO}_{x}$ film on $\mathrm{Si}$ (100). Tip is grounded, positive (left column) and negative (right) sample biases are shown. (a) Area of positive defect, (b) area of negative defect, and (c) area of no defect.

same for these regions. The tunneling barrier was determined by a single-parameter fit to the Simmons ${ }^{7}$ tunneling model to be $3.4 \mathrm{eV}$. Since area $\mathrm{C}$ exhibits no defect states in the film, it may be used as reference for determining the position of the Fermi level of the film relative to the oxides band gap, i.e., the Fermi level lies $3.4 \mathrm{eV}$ below the conduction band.

The issue of spatial resolution is addressed in Fig. 3 in which high conductance regions outline defects. A cross section of the current map over a midsized defect illustrates the lateral information density. The defect feature appears to be 2-3 nm in diameter with several data points within this range. Note also that smaller defects are also resolved. Therefore the limit in defect detection is indeed $1 \mathrm{~nm}$ or even smaller, similar to the observations of Bussmann and Williams ${ }^{4}$ and Eyben et al. ${ }^{2,6}$ This dimension is clearly smaller than that of the tip. The contact radius is estimated to be $4-5 \mathrm{~nm}$ using a Hertzian model and the following parameters: tip radius $=32 \mathrm{~nm}, E_{\mathrm{Si}}=170 \mathrm{GPa}, \nu_{\mathrm{Si}}=0.27, E_{\mathrm{PtIr}}$ $=200 \mathrm{GPa}, \nu_{\mathrm{PtIr}}=0.1$, and applied load $=375 \mathrm{nN}$. The calcu-

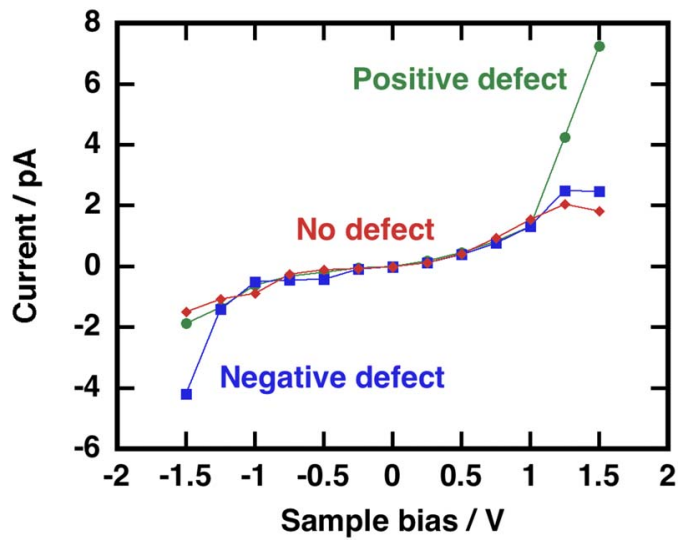

FIG. 2. (Color online) $I-V$ curves of the positive defect (area A) in Fig. 1, negative defect (area B), and no defect (area C).
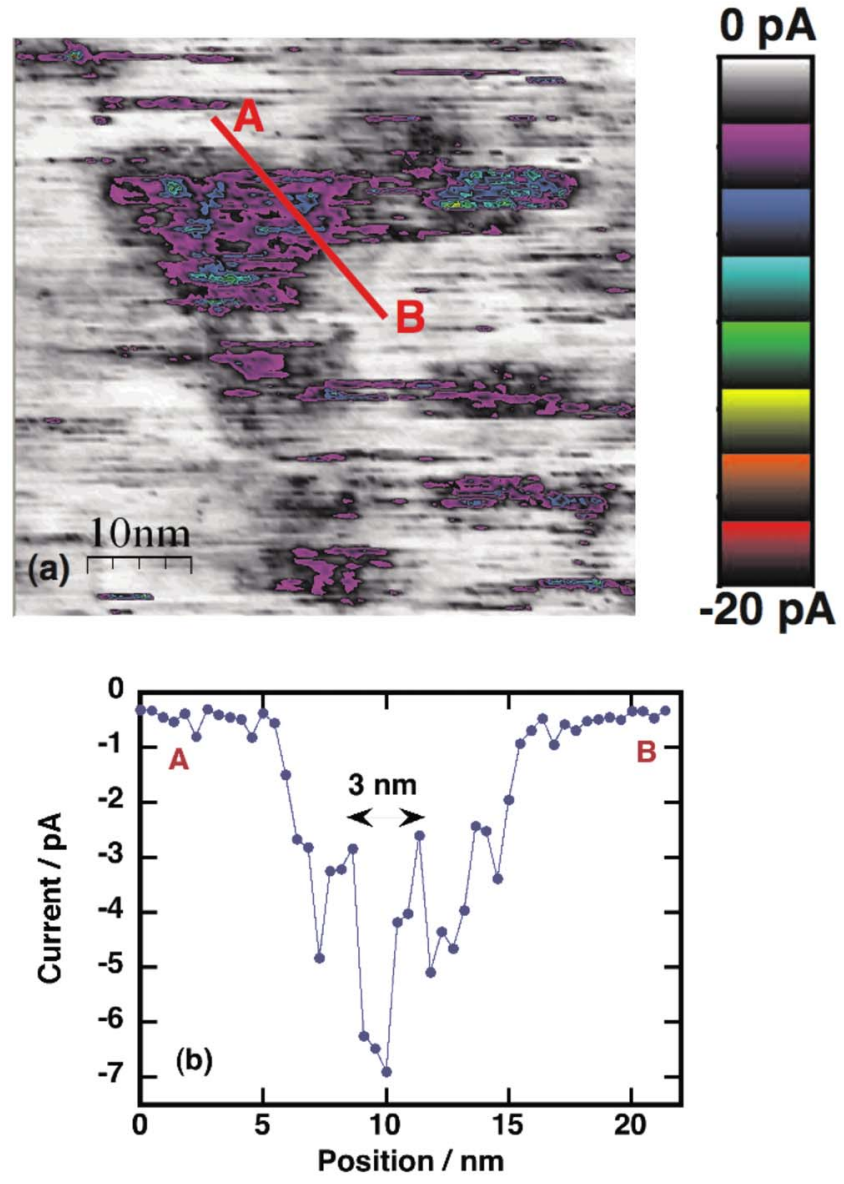

FIG. 3. (Color online) (a) Current map of $\mathrm{HfO}_{x}$ film on $\mathrm{Si}$ (100) at $-0.5 \mathrm{~V}$ bias. (b) Sections A and B showing measurement resolution better than 3 $\mathrm{nm}$.

lations ignore tip-sample adhesive force, which is small compared to the applied load; they also assume that the $20 \mathrm{~nm}$ PtIr undergoes all of the tip deformation, while the Si substrate underneath is rigid.

The reduction in the tip-surface interaction size from the contact diameter by a factor of 8-10 implies the operation of a field focusing mechanism. Two potential focusing effects are a localized phase transformation or an elastic strain induced conductivity increase. The stress distribution underneath the tip-sample junction is not uniform, with a region with half of the contact zone experiencing $50 \%$ greater stress than the nominal compressive load. ${ }^{8}$ Consequently, the load at the tip apex may be sufficiently large to induce changes in the substrate that will be restricted to a small volume under the tip. At moderately high loads a strain-induced decrease in band gap could occur, as has been quantified in macroscopic measurements. Paul and Pearson 9 reported that $\partial E_{g} / \partial P$ $=15 \mathrm{meV} / \mathrm{GPa}$ for $\mathrm{Si}$. For the $\sim 10 \mathrm{GPa}$ load used here the result is a decrease of $0.15 \mathrm{eV}$ or about $10 \%$ of the $\mathrm{Si}$ band gap. Since tunneling current is a sensitive function of $E_{g}$, this effect could be significant and is also a function of substrate dopant concentration. At higher pressures Si undergoes a phase transition from semiconducting diamondlike fcc to conductive tetragonal $\beta \mathrm{Sn}$ and then to an insulating bcc phase upon release of pressure. Shimomura et al. ${ }^{10}$ observed a sharp change in the crystalline structure of $\mathrm{Si}$ at $15 \mathrm{GPa}$, while subsequent studies placed the transition at slightly lower pressure: $10-13 \mathrm{GPa} .{ }^{11-13}$ 


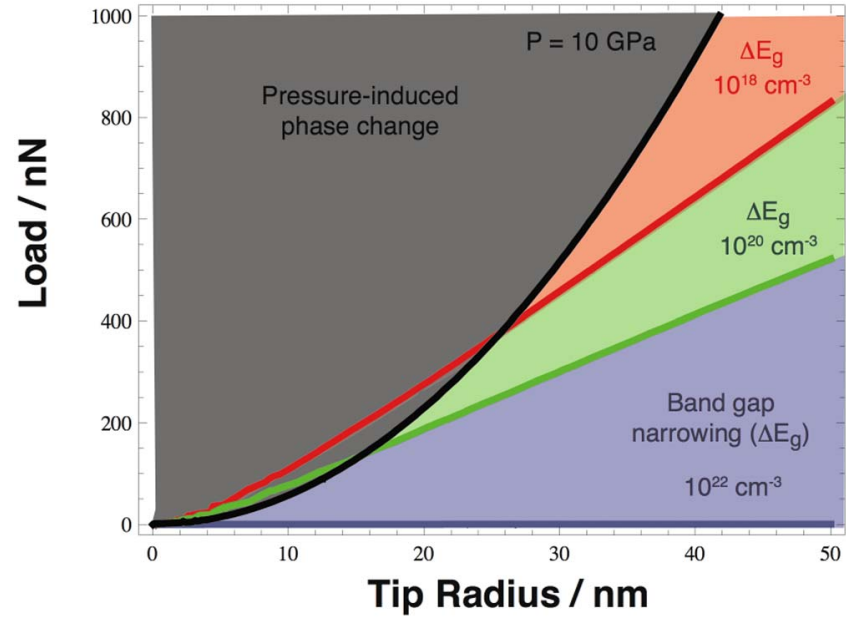

FIG. 4. (Color online) Behavior diagram showing the conditions under which phase transformation and band gap effects act to focus the tip/surface interaction, increasing spatial resolution of detection. Gray region (stress $>10 \mathrm{GPa}$ ) represents the change in conductive phase. Red, green, and blue lines indicate threshold conditions for selected doping levels of silicon at which band gap effects are detected.

To illustrate the experimental conditions under which these two mechanisms can operate, the local pressure as a function of tip size and applied load are related to limits for increased current due to band gap effects and for a local phase transformation. The band gap effect was calculated as follows. The limit of the detectable increase in current is taken to be $1 \mathrm{pA}$ based on the noise limits in the circuit. The difference in band gap that would cause a detectable current increase is calculated in the context of standard tunneling conditions, ${ }^{14}$ and the pressure necessary to reach this limit is plotted in Fig. 4.

Since the conductance depends on dopant concentration, the limits over the four orders of magnitude typical of devices are presented. Superimposed on this "behavior diagram" is the solution for load at which the phase transforma- tion occurs. The behavior diagram in Fig. 4 shows the regime of tip size and applied load where the phase transformation occurs. Under these conditions, the highly conductive transformed region under the tip becomes the effective probe area, reducing the interaction area by a factor of 4 given the stress gradients beneath the tip. The lower pressure conditions under which the band gap effect results in increased current are also shown. Here the stress gradient results in a continuous conductivity gradient that is focused somewhat less sharply. Both mechanisms result in the localization of the probe area and can account for the increased spatial resolution.

This research was supported by the Department of Energy, Office of Basic Energy Science (Grant No. DE-FG0200ER45813) and Intel Corporation. The use of facilities in the UPenn Nano/Bio Interface Center is acknowledged.

${ }^{1}$ Scanning Probe Microscopy and Spectroscopy: Theory, Techniques, and Applications, 2nd ed., edited by D. A. Bonnell (Wiley, New York, 2001).

${ }^{2}$ P. Eyben, D. Alvarez, M. Jurczak, R. Rooyackers, A. De Keersgieter, E. Augendre, and W. Vandervorst, J. Vac. Sci. Technol. B 22, 364 (2004).

${ }^{3}$ S. E. Park, N. V. Nguyen, J. J. Kopanski, J. S. Suehle, and E. M. Vogel, J. Vac. Sci. Technol. B 24, 404 (2006).

${ }^{4}$ E. Bussmann and C. C. Williams, Rev. Sci. Instrum. 75, 422 (2004).

${ }^{5}$ O. Douheret, S. Bonsels, and S. Anand, J. Vac. Sci. Technol. B 23, 61 (2005)

${ }^{6}$ P. Eyben, T. Janssens, and W. Vandervorst, Mater. Sci. Eng., B 124-125, 45 (2005).

${ }^{7}$ J. G. Simmons, J. Appl. Phys. 34, 2581 (1963).

${ }^{8}$ N. Schwarzer, F. Richter, and G. Hecht, Surf. Coat. Technol. 114, 292 (1999).

${ }^{9}$ W. Paul and G. L. Pearson, Phys. Rev. 98, 1755 (1955).

${ }^{10}$ O. Shimomura, S. Minomura, N. Sakai, K. Asaumi, K. Tamura, J. Fukushima, and H. Endo, Philos. Mag. 29, 547 (1974).

${ }^{11}$ D. R. Clarke, M. C. Kroll, P. D. Kirchner, R. F. Cook, and B. J. Hockey, Phys. Rev. Lett. 60, 2156 (1988).

${ }^{12}$ R. J. Needs and A. Mujica, Phys. Rev. B 51, 9652 (1995).

${ }^{13}$ Y. H. Lin, S. R. Jian, Y. S. Lai, and P. F. Yang, Nanoscale Res. Lett. 3, 71 (2008).

${ }^{14}$ S. M. Sze, Physics of Semiconductor Devices, 2nd ed. (Wiley, New York, 1981). 